\title{
S-ICD: Is it time for radiological follow-ups?
}

\author{
Mariano Rillo ${ }^{1}$, Zefferino Palamà ${ }^{2}$, Giulia $\mathrm{My}^{2}$, Raffaele Punzi ${ }^{3}$, Aurelio Andrea ${ }^{1}$, Angelo \\ Aloisio $^{1}$, Cesare Giannattasio ${ }^{1}$, and Luigi $\mathrm{My}^{1}$ \\ ${ }^{1}$ Casa Di Cura Villa Verde \\ ${ }^{2}$ Casa di Cura Villa Verde Srl \\ ${ }^{3}$ Casa di Cura Villa Verde
}

March 4, 2021

\section{S-ICD: Is it time for radiological follow-ups?}

Short title: S-ICD lead migration

Mariano Rillo M.D. ${ }^{1}$, Zefferino Palamà M.D. ${ }^{1}$, Giulia My M.D. ${ }^{2}$, Raffaele Punzi M.D. ${ }^{1}$, Andrea Aurelio M.D. ${ }^{2}$, Angelo Aloisio M.D. ${ }^{2}$, Cesare Giannattasio M.D. ${ }^{2}$, Luigi My M.D. ${ }^{2}$

Electrophysiology Service, Division of Cardiology - Casa di Cura Villa Verde, Taranto, Italy

Cardiology Unit - Casa di Cura "Villa Verde", Taranto, Italy

Corresponding author:

Zefferino Palamà Casa di Cura "Villa Verde"- Taranto- Italy Via Golfo di Taranto, 22- Taranto Email:zefferino.palama@icloud.com

All data are avilable in EP lab in casa di Cura Villa Verde, Taranto, Italy.

No funding resources to declare.

Conflict of interest: none.

Keywords: Subcutaneous ICD, S-ICD lead migration, ICD lead complication

\section{Abstract}

We present the case of an asymptomatic S-ICD lead migration in a 38-years-old woman, previously implanted in 2013 (3 incision technique, subcutaneous pocket, secondary prevention) and subsequent need of MRI compatible device. During the new procedure, due to lack of the thoracic lead detection at previous scars we documented fluoroscopically its migration down and left lateral. The possibility of lead migration should be considered because an important alteration in its position position could affect defibrillation threshold. A periodic chest X-rays compared to chest X-ray that performed post implantation could be method of screening in addition to electronic follow-ups, especially before S-ICD replacement.

Introduction

The implantable cardioverter-defibrillator (ICD) is the cornerstone in sudden death prevention ${ }^{1}$. The subcutaneous ICD (S-ICD) is an alternative to the traditional transvenous ICD (TV-ICD) without the risk of endovascular lead complications. It demonstrated no significant difference in overall and arrhythmic mortality rates but a significant reduction in device-related complications with only $2 / 426$ lead repositionings ${ }^{2}$.

Clinical case 
A 38-years-old woman was admitted in 2013 because of a sustained polymorphic ventricular tachycardia, with a significant rise of troponin values and ECG signs of ischemia. The patient is also affected by gynoid obesity (body mass index $33 \mathrm{~kg} / \mathrm{m} 2$ ). Echocardiography imaging showed a severe impairment of systolic left ventricular function (LVEF: 30\%), but we found normal coronaries at coronary angiography. Cardiac magnetic resonance imaging showed late gadolinium enhancement in inferior wall, therefore a diagnosis of myocarditis was made with severe systolic left ventricular dysfunction. After a successful surface electrocardiogram (ECG) screening, the patient underwent S-ICD implantation (SQ-RX Pulse Generator Model 1010 and Q-TRAK Subcutaneous Electrode Model 3010, Cameron Health/Boston Scientific, San Clemente, CA). The generator pocket was created in the midaxillary line superficial to the latissimus dorsi between the fifth and sixth intercostal spaces and the electrode along the left parasternal border. As the technique of the time, the lead was also fixed at the level of the II intercostal space over manubrio sternal junction, (3incision technique). All sutures were adhered to the fascia of the sternum. At implant, ventricular detection test showed appropriate sensing in primary and alternate vectors. The post implantation defibrillation test (DFT) showed a defibrillation threshold $<65$ Joule. Post implantation chest X-ray showed that the generator was positioned anteriorly to the midaxillary line with a correct lead positioning. Post-operative course was uneventful, and the patient was discharged from the hospital 2 days after.

At routine follow-ups good paramethers were found with no need to change sensing vectors. In July 2020 the patient was scheduled for S-ICD replacement due to battery depletion (3 non treated non sustained ventricular tachycardia in event memory, $81 \mathrm{ohm}$ lead impedance). Echocardiography imaging showed a partial recovery of systolic left ventricular function (LVEF: 45\%). The patient was also scheduled for a brain MRI for a meningioma follow up. The lead implanted in 2013 was found not to be MRI compatible and for this reason the entire system was extracted and a fully MRI compatible device was reimplanted.

At surgical exploration the device was fixed and sitting anterior to the latissimus dorsi muscle but during the lead extraction procedure it appeared impossible to find the lead anchor points on sleeves both in the upper parasternal region (manubrio sternal junction) and in the xiphoid region. The radiological exploration allowed to highlight the displacement of the lead below the manubrio sternal junction scar about $5 \mathrm{~cm}$ (figure 1 , klemmer forceps used as landmark of the implant scar) and the left lateral displacement of the entire coil also in the xyphoid region, so as not to completely embrace the entire cardiac shadow (figure 2). The ICD lead was extracted and the 1-0 silk retention appeared to be frayed and broken, as as a result of a traction or sliding of the entire lead which tore down both the manubrio sternal junction retention and that in the xiphoid region.

The new fully MRI compatible device was implanted (EMBLEM MRI S-ICD Pulse Generator A219 Boston Scientific and SQ1 Emblem S-ICD 3501-45 cm) with a 3-incision technique (in order to ensure a solid fixation of the distal lead tip). It was positioned in a new intermuscular pocket position by blunt dissection between the posterior surface of the latissimus dorsi muscle and the anterior surface of the serratus anterior muscle to have the pocket in the virtual anatomical space between the two muscles. The post implantation ventricular detection test showed appropriate sensing in primary and alternate vectors and the defibrillation test showed a defibrillation threshold $<65$ Joule. Post-operative course was uneventful, and the patient was discharged from the hospital after 2 days. X-ray examination showed a correct lead positioning at discharge (figure 3).

Question: Would the old device deliver effective therapy for malignant ventricular arrhythmia?

\section{Discussion}

Totally subcutaneous ICD (S-ICD) is designed to afford the same life-saving benefit of the conventional transvenous (TV) ICDs while avoiding the shortcomings of the TV-leads and to simplifying the implant techniques and hence expanding the use of ICDs in clinical practice. Both the EFFORTLESS REGISTRY ${ }^{3}$ at first and the PRAETORIAN trial ${ }^{2}$ demonstrated appropriate system performance with clinical event rates and inappropriate shock rates comparable with those reported for conventional ICDs. S-ICD lead dislodgment rates are very low and clinically relevant as associated with inappropriate shocks due to sensing alterations ${ }^{4}$. 
Answer: Our case is the first that describes a partial lead migration without altering the sensing parameters. To date, few cases of displacement have been recorded to clearly define in literature it risk factors. Probably the patient's obesity and her young age (as well as in sports athletes) represented breeding grounds for the subcutaneous lead migration. The routine follow-ups done over the years have never detected the dislocation, but we cannot know if the lead was in a suitable position to guarantee an effective shock in case of ventricular fibrillation. In fact it is shown by post S-ICD implant defibrillation threshold tests that a slightly more lateral or more immersed in fat position can significantly affect this threshold, with the need to immediately reposition the lead $\mathrm{d}^{5-7}$. Also, the need to extract the lead to perform the brain MRI allowed to clearly reveal the displacement. The limit in our case report is represented by the non-induction of VF before the replacement of the entire S-ICD system to verify the effectiveness or otherwise of the shock and it is justified by the unethicality of this approach, as a new induction was carried out for the evaluation of the final defibrillation thresholds. The absence of inappropriate shocks during the follow-up can be explained by preserved sensing and the absence of appropriate shocks by the absence of ventricular tachyarrhythmias. In fact, after the first implant only 3 non sustained ventricular tachycardia were detected (there was also a partial recovery in ejection fraction).

Teaching points and proposed solution

The S-ICD represents a cornerstone in the sudden death prevention in young subjects. However, due to the characteristics of these patients, the possibility of lead migration (often asymptomatic and not diagnosable with the device electronic check) must be considered in the S-ICD follow up program, especially in obese patients or sports athletes. Indeed, an important alteration in lead position could affect the defibrillation threshold. At this point we may need to answer a question: Is it possible to demonstrate only with electronic control in the follow up that S-ICDs are sufficiently effective? In fact, it is very difficult to appreciate changes in the EGMs amplitude at an ordinary follow-up. Probably, in subjects at higher risk of dislocation a periodic (or at least one in the first months after implantation) chest x-ray to be compared to the one performed post implantation could represent a means of screening to be added to the electronic follow-ups. Data regarding DFT testing in S-ICD patients undergoing its replacement is scarce and it is unknow how many physicians perform DFT testing after its replacement ${ }^{8}$. Current guidelines recommend DFT testing in patients undergoing S-ICD implantation but do not indicate DFT after replacement. Surely this indication could be mandatory at the time of S-ICD replacement, in case of suspected partial dislocation diagnosed by chest x-ray.

\section{References}

1. Pick JM, Batra AS. Implantable cardioverter-defibrillator implantation for primary and secondary prevention: indications and outcomes. Cardiol Young . 2017 Jan;27(S1):S126-S131. doi: $10.1017 / \mathrm{S} 1047951116002365$.

2. Knops RE, Olde Nordkamp LRA, Delnoy PP et al. Subcutaneous or Transvenous Defibrillator Therapy (PRAETORIAN TRIAL). N Engl J Med 2020;383:526-36. doi: 10.1056/NEJMoa1915932.

3. Lambiase PD, Barr C, Theuns DAMJ et al. Worldwide experience with a totally subcutaneous implantable defibrillator: early results from the EFFORTLESS S-ICD Registry. European Heart Journal, Volume 35, Issue 25, 1 July 2014, Pages 1657-1665,https://doi.org/10.1093/eurheartj/ehu112

4. Kiamanesh O, Larsen JM, Bashir J et al. Mobile subcutaneous implantable cardioverter defibrillator leads to oversensing and inappropriate shocks. Heart Rhythm Case Reports 2019;5:371373https://doi.org/10.1016/j.hrcr.2019.04.001

5. Hirao T, Nitta J, Sato A, Takahashi Y, Goya M, Hirao K. High defibrillation threshold with a subcutaneous implantable cardiac defibrillator due to the lead having been positioned in the fat layer. Case Reports J Arrhythm. 2018 Feb 8;34(2):198-200. doi: 10.1002/joa3.12033.

6. Sugumar H, Tung MK, Lee AJ, Bashir J, Lane C. Subcutaneous ICD lead position affects defibrillation threshold. Heart Rhythm Case Reports, Vol 4, No 1, January 2018. doi: https://doi.org/10.1016/j.hrcr.2017.10.017.

7. 7. Heist EK, Belalcazar A, Stahl W, Brouwer TF, Knops RE. Determinants of Subcutaneous Implata- 
ble Cardioverter-Defibrillator Efficacy: A Computer Modeling Study. JACC Clin Electrophysiolo.2017 Apr;3(4):405-414. doi: 10.1016/j.jacep.2016.10.016.

8. Rudic B, Tulumen E, Fastenrath F, Akin I, Borggrefe M, Kuschyk J. Defibrillation failure in patients undergoing replacement of subcutaneous defibrillator pulse generator. Heart Rhythm. 2020 Mar;17(3):455459. doi: 10.1016/j.hrthm.2019.10.024.

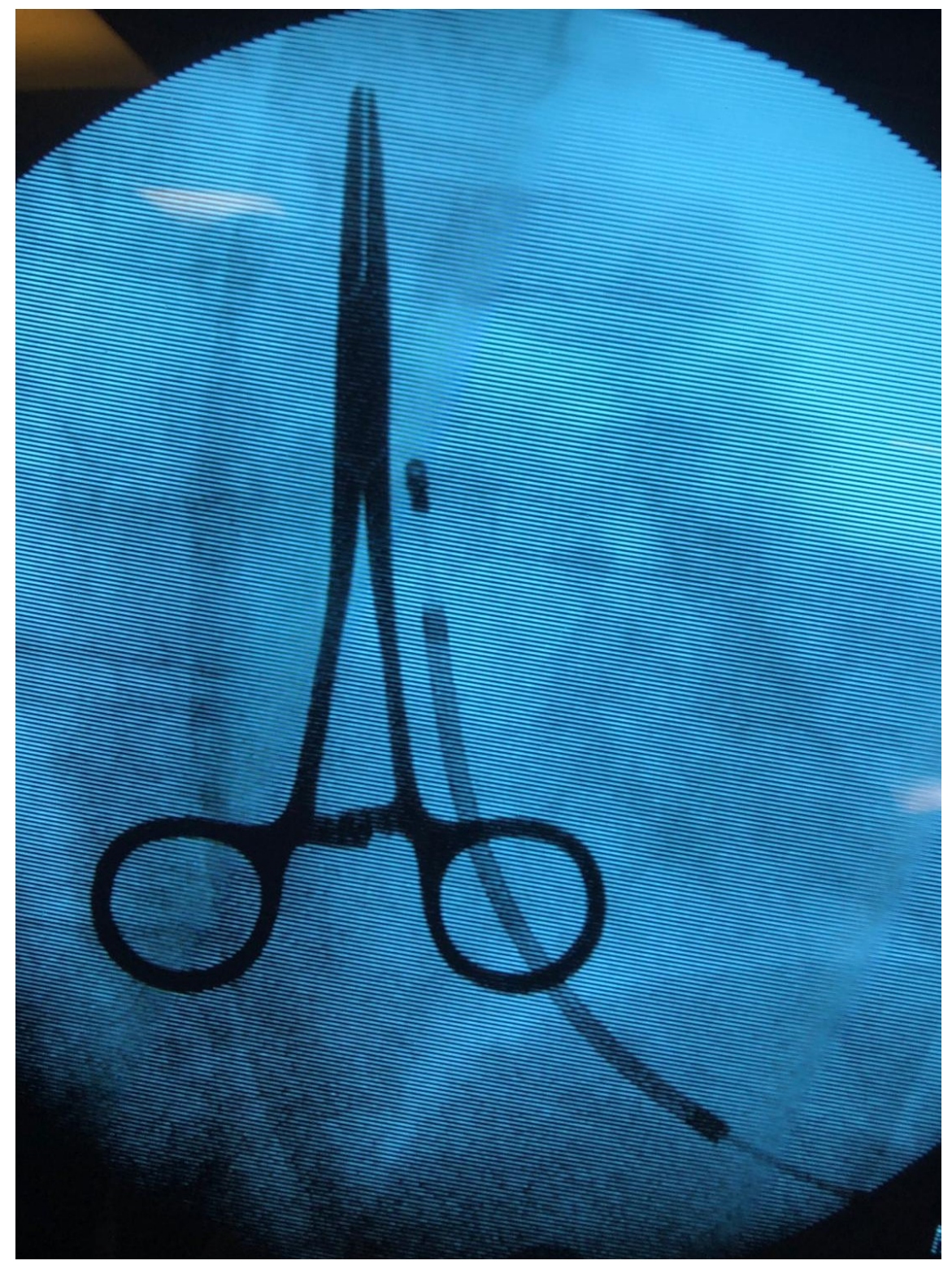

Figure 1: X-ay showing lead tip migration below the manubrio sternal junction scar about $5 \mathrm{~cm}$, klemmer forceps used as landmark of the implant scar. 


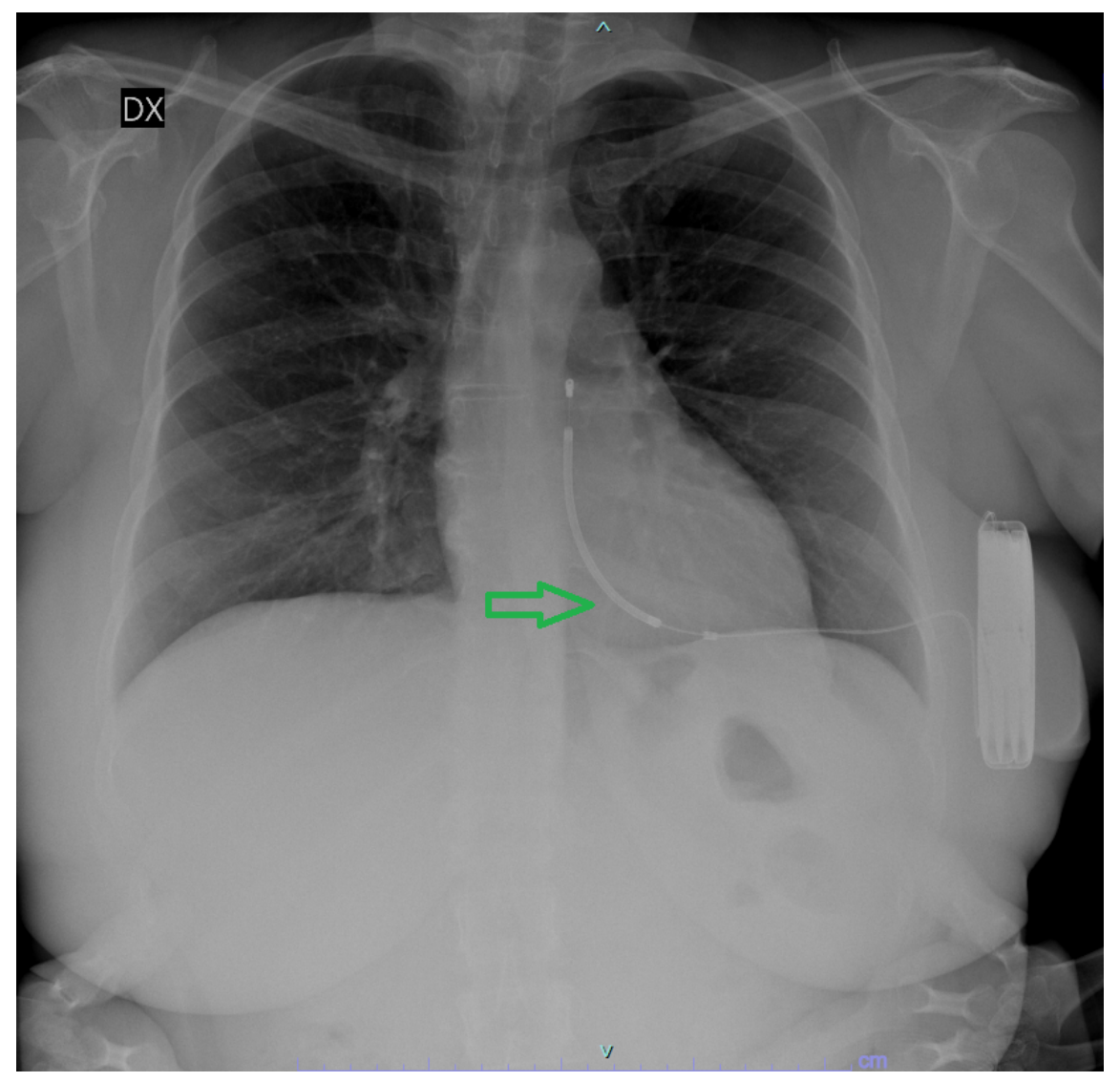

Figure 2: X-ray showing left lateral displacement of the entire coil so as not to completely embrace the entire cardiac shadow (arrows shows lead migration). 


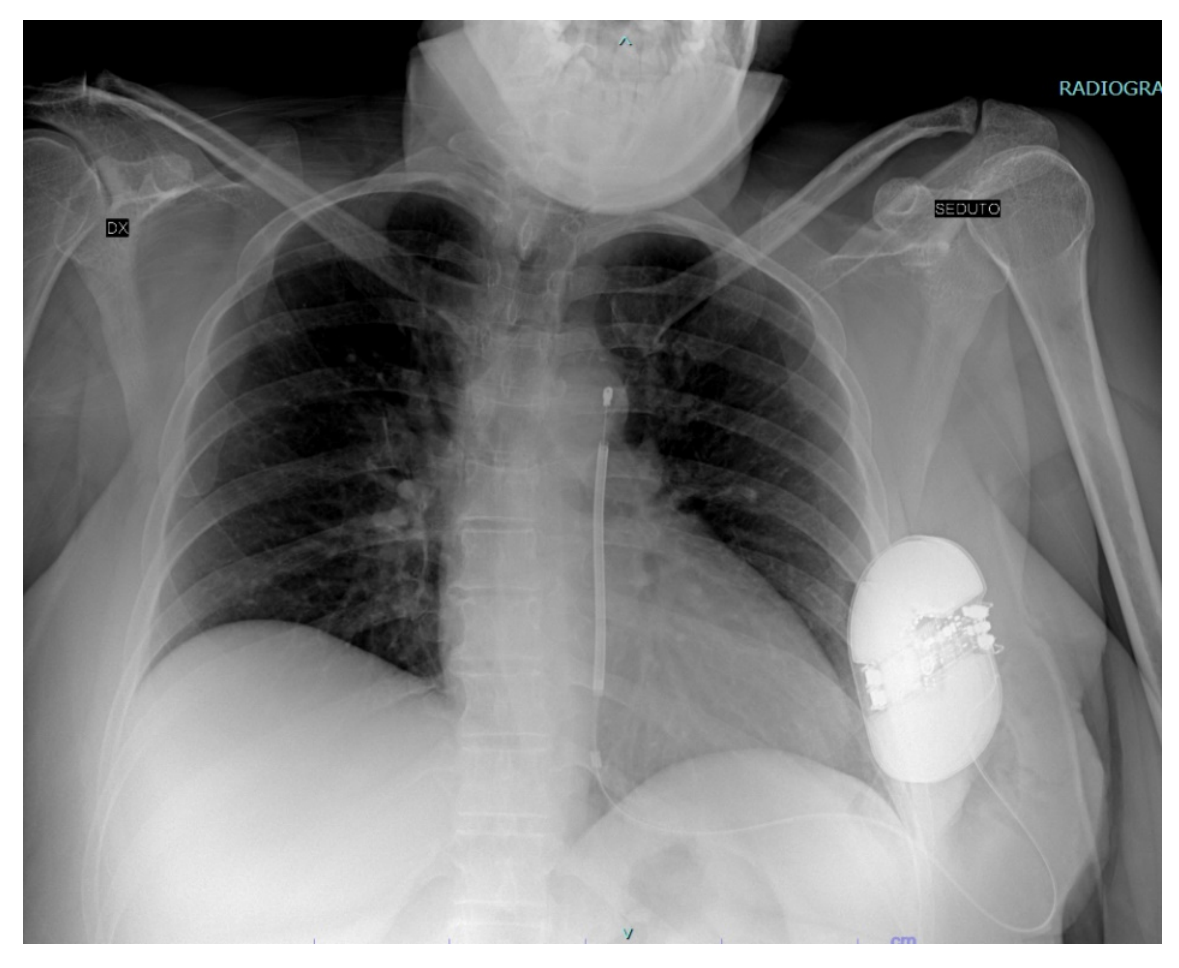

Figure 3: Post implant X-ray showing correct lead positioning. 